\title{
Packing and covering a unit equilateral triangle with equilateral triangles
}

Yuqin Zhang ${ }^{1} \quad$ Yonghui Fan ${ }^{2 *}$

${ }^{1}$ Department of Mathematics

Beijing Institute of Technology, 100081, Beijing, China

email: yuqinzhang@126.com

${ }^{2}$ College of Mathematics and Information Science

Hebei Normal University, 050016, Shijiazhuang, China

Submitted: Jun 7, 2005 ; Accepted: Oct 20, 2005; Published: Oct 25, 2005

\begin{abstract}
Packing and covering are elementary but very important in combinatorial geometry, they have great practical and theoretical significance. In this paper, we discuss a problem on packing and covering a unit equilateral triangle with smaller triangles which is originated from one of Erdös' favorite problems.
\end{abstract}

Keywords: packing, minimal covering

Mathematics Subject Classification (2000): 52C15

\section{Introduction}

Packing and covering are elementary but very important in combinatorial geometry, they have great practical and theoretical significance. In 1932, Erdös posed one of his favorite problems on square-packing which was included in [2]: Let $S$ be a unit square. Inscribe $n$ squares with no common interior point. Denote by $e_{1}, e_{2}, \ldots, e_{n}$ the sides length of these squares. Put $f(n)=\max \sum_{i=1}^{n} e_{i}$. In [3], P. Erdős and Soifer gave some results of $f(n)$.

In [1], Connie Campbell and William Staton considered this problem again. Because packing and covering are usually dual to each other, we discussed a problem of a minimal square-covering in [5]. In this paper, we generalize this kind of problem to the case of using equilateral triangles to pack and cover a unit equilateral triangle, and obtain corresponding results.

\footnotetext{
*Foundation items: This work is supported by the Doctoral Funds of Hebei Province in China (B2004114).
} 


\section{$2 \quad$ Packing a unit equilateral triangle}

Firstly, we give the definition of the packing function:

Definition 2.1. Let $T$ be a unit equilateral triangle. Inscribe $n$ equilateral triangles $T_{1}, T_{2}, \ldots, T_{n}$ with no common interior point in such a way which satisfies: $T_{i}$ has side of length $t_{i}\left(0<t_{i} \leq 1\right)$ and is placed so that at least one of its sides is parallel to that of $T$.

Define $t(n)=\max \sum_{i=1}^{n} t_{i}$.

In this part, we mainly exploit the method of [1] to get the bounds of $t(n)$ and obtain a corresponding result. Here we list some of the proofs so that the readers may better understand.

Theorem 2.2. The following estimates are true for all positive integers $n$ :

(1) $t(n) \leq \sqrt{n}$.

(2) $t(n) \leq t(n+1)$.

(3) $t(n)<t(n+2)$.

Proof. (1)Let $\mathbf{s}$ be the vector $\left(t_{1}, t_{2}, \ldots, t_{n}\right)$, where the $t_{i}$ denote the length of the sides of the equilateral triangles in the packing, and let $\mathbf{v}$ be the vector $(1,1, \ldots, 1)$. Now $\sum_{i=1}^{n} t_{i} \leq\|\mathbf{s}\|\|\mathbf{v}\| \leq \sum_{i=1}^{n} t_{i}^{2} n^{\frac{1}{2}}=\frac{2}{\sqrt{3}} \sum_{i=1}^{n}\left(\frac{\sqrt{3}}{2} t_{i}^{2}\right) n^{\frac{1}{2}} \leq n^{\frac{1}{2}}$.

It's easy to get (2),(3) by replacing a $T_{i}$ with 2 or 3 equilateral triangles with sides of length $\frac{t_{i}}{2}$.

Definition 2.3. For a equilateral triangle $T$, dissect each of its 3 sides into $n$ equal parts, then through these dissecting points draw parallel lines of the sides of $T$, so we get a packing of $T$ by $n^{2}$ equilateral triangles with sides of length $\frac{1}{n}$. Such a configuration is called an $n^{2}$-grid. When $T$ is a unit equilateral triangle, the packing is a standard $n^{2}$-packing.

See Figure 1 for the case $n=3$.

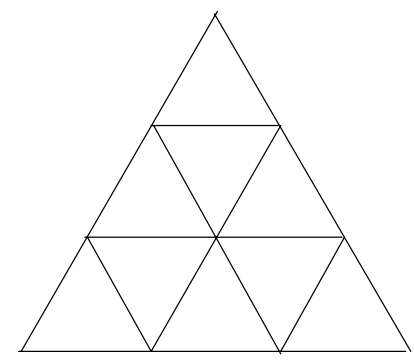

Figure 1: a $3^{2}$-grid

Proposition 2.4. $t\left(k^{2}\right)=k$. 
Proof. By Definition 2.3, it's easy to know that for the standard $k^{2}$-packing, $n=k^{2}, t_{i}=\frac{1}{k}$ and $\sum_{i=1}^{n} t_{i}=\frac{1}{k} k^{2}=k$. So by the Definition of $t(n), t\left(k^{2}\right) \geq k$ which along with Theorem 2.2(1) provides the desired equality.

Proposition 2.5. For $k \geq 2, t\left(k^{2}-1\right) \geq k-\frac{1}{k}$.

Proof. Consider the standard $k^{2}$-packing with one equilateral triangle removed.

Theorem 2.6. If $n$ is a positive integer such that $(n-1)$ is not a perfect square number, then $t(n)>(n-1)^{\frac{1}{2}}$.

Proof. When $n=k^{2}$, by Proposition 2.4, $t(n)=\sqrt{n}>\sqrt{n-1}$.

When $n=k^{2}-1$, by Proposition 2.5, $t^{2}(n) \geq\left(k-\frac{1}{k}\right)^{2}=k^{2}-1-1+\frac{1}{k^{2}}>n-1$. That is $t(n)>\sqrt{n-1}$.

When $n \neq k^{2}, k$ must lie between two perfect square numbers of different parity. That is, there is an integer $k$ such that $k^{2}<n<(k+1)^{2}, n-k^{2}$ and $(k+1)^{2}-n$ have different parity. When neither $n-1$ nor $n+1$ is a perfect square number, consider the values of $n$ where $k^{2}+1<n<(k+1)^{2}-1$, there are two cases which provide the lower bound of $t(n)$ for all $n$ on the interval $\left[k^{2}+2,(k+1)^{2}-2\right]$ :

Case 1. $(k+1)^{2}-n$ is odd. Say, $(k+1)^{2}-n=2 a+1(a \geq 1), k^{2}<n \leq(k+1)^{2}-3$. From a standard $(k+1)^{2}$-packing of $T$, remove an $(a+1)^{2}$-grid and replace it with an $a^{2}$-grid packing the same area. The result is a packing of $(k+1)^{2}-(a+1)^{2}+a^{2}=(k+1)^{2}-2 a-1=$ $n$ equilateral triangles, the sum of whose length is $\left[(k+1)^{2}-(a+1)^{2}\right] \frac{1}{k+1}+a^{2}\left(\frac{a+1}{a}\right)\left(\frac{1}{k+1}\right)=$ $k+1-\frac{a+1}{k+1}$.

So $t(n) \geq k+1-\frac{a+1}{k+1}, t^{2}(n) \geq\left(k+1-\frac{a+1}{k+1}\right)^{2}=(k+1)^{2}-2 a-1+\left(\frac{a+1}{k+1}\right)^{2}-1>n-1$. That is, $t(n)>\sqrt{n-1}$.

Case 2. $n-k^{2}$ is odd. Say, $n-k^{2}=2 a-1(a \geq 2), k^{2}+3 \leq n<(k+1)^{2}$. From a standard $k^{2}$-packing of $T$, remove an $(a-1)^{2}$-grid and replace it with an $a^{2}$-grid covering the same area. The result is a packing of $k^{2}-(a-1)^{2}+a^{2}=k^{2}+2 a-1=n$ equilateral triangles of the unit equilateral triangle $T$. The sum of the length of sides is $\left[k^{2}-(a-1)^{2}\right] \frac{1}{k}+a^{2}\left(\frac{a-1}{a}\right)\left(\frac{1}{k}\right)=k+\frac{a-1}{k}$.

So $t(n) \geq k+\frac{a-1}{k}, t(n)^{2} \geq\left(k+\frac{a-1}{k}\right)^{2}=k^{2}+2 a-1+\left(\frac{a-1}{k}\right)^{2}-1>n-1$. That is, $t(n)>\sqrt{n-1}$.

Similar to [1], by Theorem 2.6, we can easily get the following result.

Theorem 2.7. If $t(n+1)=t(n)$, then $n$ is a perfect square number.

On the other hand, we think the following is right:

Conjecture 2.8. $t\left(n^{2}+1\right)=t\left(n^{2}\right)$. 


\section{Covering a unit equilateral triangle}

Definition 3.1. Let $T$ be a unit equilateral triangle. If $n$ equilateral triangles $T_{1}, T_{2}, \ldots, T_{n}$ can cover $T$ in such a way which satisfies:

(1) $T_{i}$ has side of length $t_{i}\left(0<t_{i}<1\right)$ and is placed so that at least one of its sides is parallel to that of $T$;

(2) $T_{i}$ can't be smaller, that is, there doesn't exist any $T_{i 1} \subset T_{i}$ such that $\left\{T_{j}, j=\right.$ $1,2, \ldots, i-1, i+1, \ldots, n\} \cup\left\{T_{i 1}\right\}$ can cover $T$. (Here we admit translation.)

We call this kind of covering a minimal covering.

In the meaning of the minimal covering, define:

$T_{1}(n)=\min \sum_{i=1}^{n} t_{i}, T_{2}(n)=\max \sum_{i=1}^{n} t_{i}$.

When $n \leq 2$, since $0<t_{i}<1$, each $T_{i}(i=1,2)$ can only cover one corner of a unit equilateral triangle, but it has three corners, so $T_{1}, T_{2}$ can't cover $T$. That is, when $n \leq 2$, $T_{i}(n)(i=1,2)$ has no meaning. So in the following, let $n \geq 3$.

\subsection{The upper bound of $T_{1}(n)$}

Theorem 3.2. When $n$ is even, $T_{1}(n) \leq 3-\frac{4}{n}$.

Proof. Consider a covering of a unit equilateral triangle $T$ with a equilateral triangle $T_{1}$ which has side of length $x$ and $n-1$ equilateral triangles $T_{2}, T_{3}, \ldots, T_{n}$ each of which has sides of length $1-x$ such that $\frac{n}{2}(1-x)=1$, which implies $x=1-\frac{2}{n}$. When $n=6$, see Figure 2 for the placement. It's easy to see this is a minimal covering. So by the definition of $T_{1}(n), T_{1}(n) \leq x+(n-1)(1-x)=3-\frac{4}{n}$.

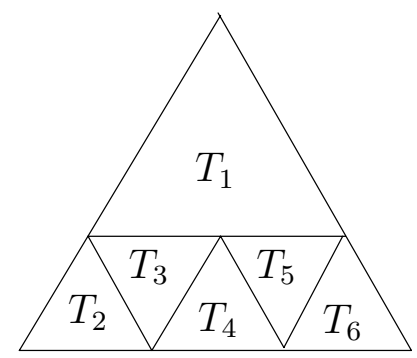

Figure 2: a unit equilateral triangle covered by six smaller equilateral triangles

Proposition 3.3. $T_{1}(3) \leq 2$.

Proof. Consider a covering of a unit equilateral triangle $T$ with 3 equilateral triangles $T_{1}, T_{2}, T_{3}$ each of which has sides of length $\frac{2}{3}$. See Figure 3 for the placement. It's easy to see this is a minimal covering. So by the definition of $T_{1}(n), T_{1}(3) \leq 3 \times \frac{2}{3}=2$. 


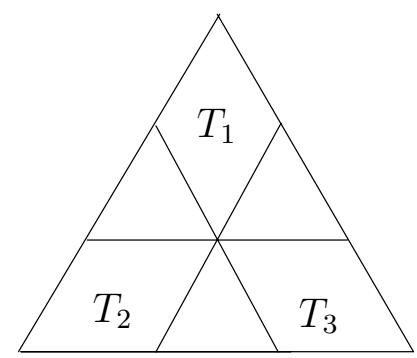

Figure 3: a unit equilateral triangle covered by 3 smaller equilateral triangles

Proposition 3.4. $T_{1}(5)<\frac{9}{4}$.

Proof. Consider a covering of a unit equilateral triangle $T$ with one equilateral triangle $T_{1}$ which has side of length $x, 2$ equilateral triangles $T_{2}, T_{3}$ each of which has sides of length $y$ and 2 equilateral triangles $T_{4}, T_{5}$ each of which has sides of length $1-x$, such that $y<2(1-x)$ and $2 y-x=\frac{x-(1-x)}{2}$, which implies $y=x-\frac{1}{4}$ and $\frac{1}{2}<x<\frac{3}{4}$. See Figure 4 for the placement. It's easy to see this is a minimal covering. So by the definition of $T_{1}(n), T_{1}(5) \leq x+2 y+2(1-x)=x+\frac{3}{2}<\frac{3}{4}+\frac{3}{2}=\frac{9}{4}$.

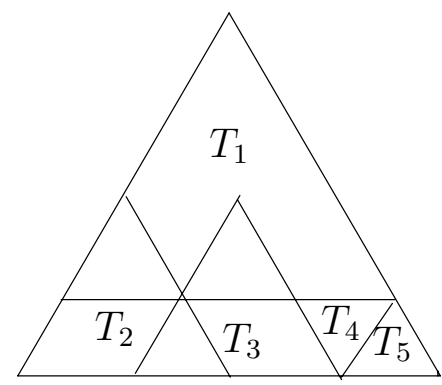

Figure 4: a unit equilateral triangle covered by 5 smaller equilateral triangles

Theorem 3.5. When $n$ is odd and $n \geq 7, T_{1}(n) \leq 4-\frac{6}{n-3}$.

Proof. Consider a covering of a unit equilateral triangle $T$ with 4 equilateral triangles $T_{1}, T_{2}, T_{3}, T_{4}$ each of which has side of length $x$ and $n-4$ equilateral triangles $T_{5}, T_{6}, \ldots, T_{n}$ each of which has sides of length $1-2 x$, such that $\frac{(n-3)(1-2 x)}{2}=1$ which implies $x=$ $\frac{1}{2}-\frac{1}{n-3}$. when $n=7$, see Figure 5 for the placement. It's easy to see this is a minimal covering. So by the definition of $T_{1}(n), T_{1}(n) \leq 4 x+(n-4)(1-2 x)=4-\frac{6}{n-3}$.

Here we can't give the lower bound of $T_{1}(n)$, but it seems obvious that the following is right:

Conjecture 3.6. $T_{1}(n) \geq 2$. 


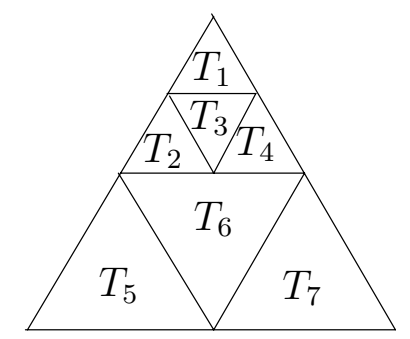

Figure 5: a unit equilateral triangle covered by seven smaller equilateral triangles

\subsection{The bounds of $T_{2}(n)$}

Proposition 3.7. $T_{2}\left(k^{2}\right) \geq k$.

Proof. It's easy to see that a standard $n$-packing is also a standard $n$-covering. By the proof of Proposition 2.4 and the definition of $T_{2}(n)$, the assertion holds.

Proposition 3.8. $T_{2}\left(k^{2}+1\right) \geq k$.

Proof. From a standard $k^{2}$-covering, remove a $2^{2}$-grid and replace it with equilateral triangles $T_{i 1}, T_{i 2}, \ldots, T_{i 5}$ covering the same area which are placed as Figure.4 such that $T_{i 1}$ is the largest equilateral triangles of $\left\{T_{i j} \mid j=1,2, \ldots, 5\right\}$ which implies that $t_{i 1} \geq \frac{1}{k}$ and $t_{i 2}=t_{i 3}=\frac{2}{k}-t_{i 1}, t_{i 4}=t_{i 5}=t_{i 1}-\frac{1}{2 k}$. The result is a covering of $k^{2}-4+5=k^{2}+1$ equilateral triangles, the sum of whose length is $t=k-\frac{4}{k}+t_{i 1}+2\left(\frac{2}{k}-t_{i 1}\right)+2\left(t_{i 1}-\frac{1}{2 k}\right)=$ $k-\frac{1}{k}+t_{i 1} \geq k$.

Obviously, any equilateral triangle of $\left\{T_{i j} \mid j=1,2, \ldots, 5\right\}$ can't be smaller. This covering is a minimal covering, so we have $T_{2}\left(k^{2}+1\right) \geq k$.

Proposition 3.9. $T_{2}\left(k^{2}-1\right) \geq k-\frac{3}{2 k}$.

Proof. From a standard $k^{2}$-covering, remove a $3^{2}$-grid and replace it with eight equilateral triangles $T_{i 1}, T_{i 2}, \ldots, T_{i 8}$ covering the same area which are placed as Figure 6 such that $T_{i 1}$ is the largest equilateral triangles of $\left\{T_{i j} \mid j=1,2, \ldots, 8\right\}$ and $t_{i 2}=t_{i 3}=t_{i 4}=t_{i 5}=t_{i 6}=$ $t_{i 7}=t_{i 8}=\frac{3}{k}-t_{i 1}$. It's obvious that $0<t_{i j}<\frac{3}{k}(j=1,2, \ldots, 8)$. And $4\left(\frac{3}{k}-t_{i 1}\right)=\frac{3}{k}$ which implies $t_{i 1}=\frac{9}{4 k}$. The result is a covering of $k^{2}-9+8=k^{2}-1$ equilateral triangles, the sum of whose length is $t=k-\frac{9}{k}+t_{i 1}+7\left(\frac{3}{k}-t_{i 1}\right)=k+\frac{12}{k}-6 t_{i 1}$. So $t \geq k+\frac{12}{k}-6 t_{i 1}=k-\frac{3}{2 k}$.

Obviously, any equilateral triangles of $\left\{T_{i j} \mid j=1,2, \ldots, 8\right\}$ can't be smaller. So any one of the resulting $k^{2}-1$ equilateral triangles can't be smaller. This covering is a minimal covering, so $T_{2}\left(k^{2}-1\right) \geq k-\frac{3}{2 k}$.

It's easy to see that a standard $n$-packing is also a standard $n$-covering. By the proof of Theorem 2.6 and the definition of $T_{2}(n)$, we can get the following result in a similar way:

Theorem 3.10. If neither $n-1$ nor $n+1$ is a perfect square number, then $T_{2}(n)>\sqrt{n-1}$. 


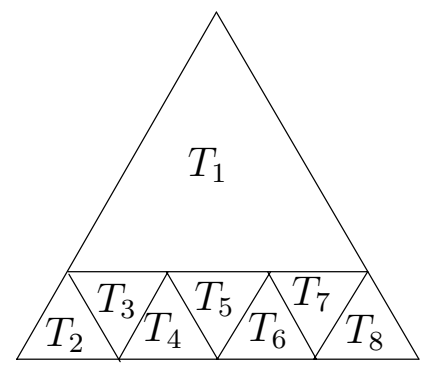

Figure 6: a $3^{2}$-grid covered by eight equilateral triangles

To get an upper bound of $T_{2}(n)$, we first list the following lemma which is a known result of [4]:

Lemma 3.11. [4] Let $T$ be a triangle and let $\left\{T_{i}\right\}_{i=1}^{n}$ be a sequence of its positive or negative copies. If the total area of $\left\{T_{i}\right\}_{i=1}^{n}$ is greater than or equal to $4|T|$ (where $|T|$ denotes the area of $T$ ), then $\left\{T_{i}\right\}_{i=1}^{n}$ permits a translative covering of $T$.

Theorem 3.12. $T_{2}(n) \leq 4 \sqrt{n}$.

Proof. Let $\left\{T_{i}\right\}_{i=1}^{n}$ be a minimal covering of the unit equilateral triangle $T$, and $t_{i}$ denote the length of the side of $T_{i}(i=1,2, \ldots, n)$. We first prove that $\sum_{i=1}^{n} \frac{\sqrt{3}}{2} t_{i}^{2} \leq 2 \sqrt{3}$. Otherwise, if $\sum_{i=1}^{n} \frac{\sqrt{3}}{2} t_{i}^{2}>2 \sqrt{3}$, there exists a $T_{i 1} \subset T_{i}$, such that $t_{i 1}<t_{i}$ and $\frac{\sqrt{3}}{2}\left(t_{i 1}^{2}+\sum_{j=1}^{i-1} t_{j}^{2}+\sum_{j=i+1}^{n} t_{j}^{2}\right) \geq$ $2 \sqrt{3}$. Notice that the area of a unit equilateral triangle is $\frac{\sqrt{3}}{2}$ and all equilateral triangle are homothetic, by Lemma 3.11, $T_{1}, T_{2}, \ldots, T_{i-1}, T_{i 1}, T_{i+1}, \ldots, T_{n}$ can cover the unit equilateral triangle $T$, which contradicts the definition of a minimal covering. So $\sum_{i=1}^{n} \frac{\sqrt{3}}{2} t_{i}^{2} \leq 2 \sqrt{3}$.

Let $\mathbf{s}$ be the vector $\left(t_{1}, t_{2}, \ldots, t_{n}\right)$, and let $\mathbf{v}$ be the vector $(1,1, \ldots, 1)$. Now $\sum_{i=1}^{n} t_{i} \leq$ $\|\mathbf{s}\|\|\mathbf{v}\| \leq \sum_{i=1}^{n} t_{i}^{2} n^{\frac{1}{2}}=\frac{2}{\sqrt{3}} n^{\frac{1}{2}} \sum_{i=1}^{n} \frac{\sqrt{3}}{2} t_{i}^{2} \leq \frac{2}{\sqrt{3}} 2 \sqrt{3} n^{\frac{1}{2}}=4 \sqrt{n}$. So $T_{2}(n) \leq 4 \sqrt{n}$.

We also have the following unsolved problem:

Problem: Improve the upper bound of $T_{2}(n)$.

\section{The case of isosceles right triangle with legs of length 1}

All the results above can be generalized to the isosceles right triangle with legs of length 1 in the same way. 


\section{Acknowledgement}

We thank the anonymous referee for a prompt, thorough reading of this paper and

for many insightful suggestions. We also would like to thank the referee for calling our attention to the paper [3].

\section{References}

[1] Connie Campbell and William Staton, A Square-packing problem of Erdős, The American Mathematical Monthly, Vol.112 (2005), 165-167.

[2] P.Erdős, Some of my favorite problems in number theory, combinatorics and geometry, Resenhas 2 (1995), 165-186.

[3] P.Erdős and Soifer, Squares in a square, Geombinatorics IV (1995), 110-114.

[4] Janusz Januszewski, Covering a triangle with sequences of its homothetic copies, Periodica Mathematica Hungarica, Vol.36(2-3) (1998), 183-189.

[5] Yuqin Zhang and Yonghui Fan, A Square-covering problem, submitted. 